\title{
Prevalence of abnormalities in knees detected by MRI in adults without knee osteoarthritis: population based observational study (Framingham Osteoarthritis Study)
}

\begin{abstract}
Objective To examine use of magnetic resonance imaging (MRI) of knees with no radiographic evidence of osteoarthritis to determine the prevalence of structural lesions associated with osteoarthritis and their relation to age, sex, and obesity.

Design Population based observational study.

Setting Community cohort in Framingham, MA, United States (Framingham osteoarthritis study).

Participants 710 people aged $>50$ who had no radiographic evidence of knee osteoarthritis (Kellgren-Lawrence grade 0 ) and who underwent MRI of the knee.

Main outcome measures Prevalence of MRI findings that are suggestive of knee osteoarthritis (osteophytes, cartilage damage, bone marrow lesions, subchondral cysts, meniscal lesions, synovitis, attrition, and ligamentous lesions) in all participants and after stratification by age, sex, body mass index (BMI), and the presence or absence of knee pain. Pain was assessed by three different questions and also by WOMAC questionnaire.

Results Of the 710 participants, 393 (55\%) were women, 660 (93\%) were white, and 206 (29\%) had knee pain in the past month. The mean age was 62.3 years and mean BMI was 27.9. Prevalence of "any abnormality" was $89 \%$ (631/710) overall. Osteophytes were the most common abnormality among all participants (74\%, 524/710), followed by cartilage damage $(69 \%, 492 / 710)$ and bone marrow lesions $(52 \%$, $371 / 710)$. The higher the age, the higher the prevalence of all types of abnormalities detectable by MRI. There were no significant differences in the prevalence of any of the features between BMI groups. The
\end{abstract}

prevalence of at least one type of pathology ("any abnormality") was high in both painful (90-97\%, depending on pain definition) and painless (86-88\%) knees.

Conclusions MRI shows lesions in the tibiofemoral joint in most middle aged and elderly people in whom knee radiographs do not show any features of osteoarthritis, regardless of pain.

\section{Introduction}

Ageing of the population and increasing obesity contribute to morbidity worldwide. Osteoarthritis is the most prevalent medically treated arthritic condition worldwide (for example, 3532 per 100000 people in the United States). ${ }^{12}$ Diagnosis of osteoarthritis is made on the basis of clinical examination or radiography. Population based longitudinal studies in the US ${ }^{3}$ and the United Kingdom ${ }^{4}$ showed the lifetime risk of knee osteoarthritis increases with age, ${ }^{3}$ with the risk highest in obese people. ${ }^{34}$ Other prevalence surveys showed that radiographic osteoarthritis of the knee is common in middle aged and older adults. ${ }^{56}$

Although many publications have reported structural changes in people with radiographic knee osteoarthritis, few data are available regarding what structural changes are present in knees without any radiographic features of osteoarthritis. About half of people with knee pain have no radiographic osteoarthritis. In clinical practice, it is unclear how to investigate and manage such people and whether additional imaging with magnetic resonance imaging would be of clinical value. Such data can be collected only in population based studies as people with normal 
knees are not usually enrolled into clinical studies or undergo further imaging evaluation. Radiography can show osteophytes, bony outgrowths at the joint margin, and narrowing of the joint space, but it cannot visualise soft tissue pathology. ${ }^{7}$ In contrast, MRI can visualise various tissues that are clinically relevant and have an important role in regard to structural progression not seen on radiography. MRI can also show incidental findings in otherwise asymptomatic people. ${ }^{89}$ In the knee, MRI visualises most components of the joint, including articular cartilage, menisci, intra-articular ligaments, synovium, bone marrow, subchondral cysts, and other periarticular and intra-articular lesions that are not detectable by radiography. ${ }^{10}$

We used MRI to evaluate the presence of structural changes in knees that were free from radiographic tibiofemoral osteoarthritis. We focused on the tibiofemoral joint, which includes numerous bony and soft tissue structures that can be evaluated by MRI. We evaluated the prevalence of cartilage damage, meniscal lesions, osteophytes, subchondral cysts, bone marrow lesions, ligamentous lesions, attrition, and synovitis on MRI in participants of the Framingham Osteoarthritis Study who had radiographically normal tibiofemoral knee joints. We also assessed whether the prevalence of these features differed according to age, sex, body mass index (BMI), or knee pain.

\section{Methods}

\section{Study design and participants}

The Framingham Community cohort was recruited from the Framingham, MA, census tract data for the year 2000 and random digit telephone dialling. All participants were examined between 2002 and 2005. This study cohort is distinct from the Framingham Heart Study and the Framingham Offspring Study cohorts. Participants were not selected on the basis of having knee or other joint problems, and potential participants were not told that knees were a focus of the study.

Eligible participants were aged at least 50 and ambulatory (the use of assistive devices such as canes and walker was permitted), with no plans to move out of the area for at least five years to accommodate the possibility of longitudinal follow-up. We excluded those with a history of bilateral total knee replacement, rheumatoid arthritis, dementia, or terminal cancer and those who had contraindications to MRI. Of 2582 people aged 50 or older and living in Framingham who were contacted by random digit dialling, 1830 expressed interest in participating in the study. ${ }^{8}$ Of those, 39 were lost to contact, 194 were ineligible for the study, and 558 declined to participate. Consequently, 1039 were examined, 993 underwent MRI, and 992 had readable scans (one knee per participant, right knee preferred; left knee if right knee not available (fig $1 \Downarrow$ ).

\section{Knee radiography and grading}

Participants underwent weight bearing posteroanterior knee radiography with the fixed-flexion protocol. ${ }^{11}$ One musculoskeletal radiologist, who was blinded to the MRI findings and clinical data, graded radiographs using the Kellgren-Lawrence grading system (intraobserver $\kappa 0.83$ ). ${ }^{12}$ Because we wanted to focus on "normal" tibiofemoral knee joints (Kellgren-Lawrence grade 0), we excluded 253 participants with radiographic tibiofemoral osteoarthritis (Kellgren-Lawrence grade 2 or above), doubtful or equivocal findings of radiographic evidence of tibiofemoral osteoarthritis (Kellgren-Lawrence grade 1), or missing radiographs or radiographic readings. Finally, we excluded 30 participants because of unreadable or poor quality MRIs. This resulted in
710 radiographically "normal" tibiofemoral knee joints being included in the final sample for analysis (fig $1 \Downarrow$ ).

\section{MRI grading of osteoarthritis features}

MRI was done with a 1.5 Tesla scanner (Siemens Medical Systems, Erlangen, Germany) with a phased array knee coil. Images from four pulse sequences were used in the assessment of osteoarthritis features: axial, sagittal and coronal fat saturated, proton density weighted, turbo spin echo images (repetition time $3610 \mathrm{msec}$; echo time $40 \mathrm{msec}$; slice thickness $3.5 \mathrm{~mm}$; interslice gap $0 \mathrm{~mm}$; echo train length 7; field of view $140 \mathrm{~mm}$ $\times 140 \mathrm{~mm}$; matrix $256 \times 256$ ) and sagittal T1 weighted spin echo images without fat saturation (repetition time $475 \mathrm{msec}$; echo time $24 \mathrm{msec}$; slice thickness $3.5 \mathrm{~mm}$; interslice gap $0 \mathrm{~mm}$; field of view $140 \mathrm{~mm} \times 140 \mathrm{~mm}$; matrix $256 \times 256$ ).

MRI scans were read by two trained and experienced musculoskeletal radiologists (who did not read the radiographs) using a standardised and validated method called the whole organ magnetic resonance imaging score (WORMS). ${ }^{14}$ They recorded the presence or absence of the specific features (described below) related to osteoarthritis that were included in our assessment of the tibiofemoral joint (that is, tibial plateaus and the central weight bearing and posterior portions of femoral condyles). In the WORMS system, the tibiofemoral joint is subdivided into 10 different subregions for scoring of each feature. Readings from all subregions were amalgamated within the knee. ${ }^{14}$ Agreement between observers ( $\kappa$ statistic) for the detection of the MRI features was as follows: cartilage damage 0.89 ; meniscal lesions 0.71 ; osteophytes 0.73 ; ligamentous lesions 0.49 ; bone marrow lesions 0.85 ; subchondral cysts 0.57 ; and synovitis 0.63 . The relatively low value of $\kappa$ for ligamentous lesions was because few knees had ligamentous lesions in the reliability sample.

Cartilage damage was considered present if there was a small focal loss less than $1 \mathrm{~cm}$ in greatest width or areas of diffuse partial or full thickness loss (WORMS grade $\geq 2$ ). In this study we did not consider intrachondral signal alterations (WORMS grade 1), which are thought to occur before cartilage damage develops ${ }^{14}$ but are of unknown clinical importance, to represent cartilage damage.

Meniscal lesions (WORMS grade $\geq 1$ ) included displaced or non-displaced meniscal tears or evidence of previous surgery (including repair and partial or complete resection) and complete maceration or destruction (that is, loss of normal contour and signal homogeneity within the meniscus) within the anterior and posterior horns and the body of the medial and lateral menisci. ${ }^{14}$

Osteophytes were considered present if there were bony projections that form along different margins of the tibiofemoral joint of the knee (WORMS grade $\geq 2$ ). Tiny bony spurs that were equivocal on visual evaluation (that is, "lipping," WORMS grade $\geq 1$ ) were not considered as osteophytes.

Ligamentous abnormalities were defined as the presence of a completely torn anterior or posterior cruciate ligament, or a torn or thickened medial or lateral collateral ligament (WORMS grade $\geq 1$ ).

Bone marrow lesions - Subchondral bone marrow lesions, also known as "bone marrow edema-like lesions," 15 were considered present if there are non-cystic subchondral areas of ill defined high signal on proton density weighted MR images with fat signal suppression (WORMS grade $\geq 1$ ).

Subchondral cysts were identified as areas of markedly increased signal intensity in the subarticular bone with sharply defined 
rounded margins and no evidence of internal marrow tissue or trabecular bone on the fat saturated proton density weighted images (WORMS grade $\geq 1$ ).

Synovitis was considered present if the synovial cavity was distended and filled with fluid (high signal intensity on fat saturated proton density weighted images), representing synovial thickening and joint effusion (WORMS grade $\geq 1$ ). ${ }^{14}$

Attrition-Flattening or depression of the articular surfaces of the tibia or femur was termed bone attrition, and any degree of deviation from the normal bony contour was considered abnormal (WORMS grade $\geq 1$ ).

\section{Additional analysis with a more stringent definition of "abnormality"}

Currently there is no concrete definition of what is "abnormal" in terms of MRI findings in the knee, and the use of different cut off points for the definition of "abnormality" might produce different results. We also examined a more stringent definition of lesions detected by MRI, which included cartilage damage and osteophytes $=$ WORMS grade $\geq 3$; all other lesions $=$ grade $\geq 2$.

\section{Assessment of weight, height, and pain}

We measured the participants' weight when they were not wearing shoes with the use of a balance beam scale and measured height with a stadiometer. At the clinic visit all participants were asked about knee symptoms with the following question: "In the past month, have you had any pain, aching, or stiffness in your knee?" (for this study, we focused on pain in the knee with MRI reading). Additionally, we assessed knee pain in three more ways. Participants responded to the questions, "Did you have knee pain lasting at least a month in the past year?" and "Do you have knee pain on most days?" A positive response to these questions was considered to indicate the presence of knee pain. Each participant was also asked to fill out the Western Ontario McMaster University arthritis index (WOMAC) questionnaire, and any score $\geq 1$ in the pain subscale in the knee was considered to indicate the presence of knee pain. For WOMAC pain, we restricted our analysis to participants who had Kellgren-Lawrence grade 0 knees bilaterally as the WOMAC questionnaire was person based and not knee based.

\section{Statistical analysis}

We calculated the prevalence of the aforementioned osteoarthritis features on MRI and stratified the data according to sex, age group (sixth decade, seventh decade, and older), BMI $(<25, \geq 25-<30, \geq 30)$, and the presence of pain. We used $\chi^{2}$ tests to assess the presence of significant differences between men and women, and among different age and BMI groups. For cartilage and bone marrow lesions, results were stratified according to the medial and lateral tibiofemoral compartments of the knee. All statistical analyses were performed with SAS for Windows, version 9.1. Results were considered to be significant when a two tailed $\mathrm{P}<0.05$.

\section{Results}

\section{Characteristics of study sample}

Of the 710 participants, 393 (55\%) were women, 660 (93\%) were white, and 206 (29\%) had painful knees. The mean age was 62.3 (range 51-89), and the mean BMI was 27.9 (range 16.6-50.6) (table $1 \Downarrow$ ).

\section{Prevalence of bony and soft tissue abnormalities on MRI with standard definition}

Overall, $631(89 \%)$ knees had at least one type of abnormality (fig $2 \Downarrow$, table $2 \Downarrow$ ). The three most common findings were osteophytes, cartilage damage, and bone marrow lesions. In the location specific analysis, cartilage damage was more prevalent in the medial tibiofemoral compartment (33\% (95\% confidence interval $30 \%$ to $37 \%), 235 / 710$ ) than in the lateral tibiofemoral compartment (20\% (17\% to $23 \%), 141 / 710)$. Likewise, there were more bone marrow lesions in the medial (19\% (16\% to $22 \%), 133 / 710)$ than in the lateral tibiofemoral compartment (12\% (10\% to $16 \%), 87 / 710)$.

Table 2 summarises the prevalence of each MRI feature overall and in men and women $\Downarrow$. The prevalence of meniscal lesions was significantly higher in men than in women (110/317 (35\%) $v 57 / 393(15 \%) ; \mathrm{P}<0.001)$. No other features were significantly different between men and women. There were no significant differences in the prevalence of any of the features between BMI groups (table 2). The prevalence of all features was within about $7 \%$ among all BMI groups.

Older age groups had more abnormalities of all types. Of the participants in their sixth decade, $86 \%(271 / 316)$ had features of osteoarthritis. The rate increased to $91 \%(227 / 249)$ in the seventh decade and 92\% (133/145) in the oldest age group. Specific types of abnormalities (cartilage damage, meniscal lesions, osteophytes, subchondral cysts) also increased with each decade (table $3 \Downarrow$ and fig $3 \Downarrow$ ). The prevalence of ligamentous lesions, bone marrow lesions, attrition, and synovial thickening and joint effusion was also higher in older age groups, but the differences between groups were not significant. The prevalence of attrition $(38 \% v 30 \% ; \mathrm{P}=0.04)$, bone marrow lesions (59\% v 50\%; $\mathrm{P}=0.03)$, and subchondral cysts $(31 \%$ v $23 \% ; \mathrm{P}=0.04$ ) was higher in participants with painful knees than those without pain (table 2). The prevalences for the other features were within about $4 \%$ of one another among painful and painless knees with no significant differences (table 2). Indeed, the prevalence of at least one type of MRI detected pathology ("any abnormality") was high in both painful (91\%) and painless $(88 \%$ ) knees (table $2 \Downarrow$ ). Regardless of the definition of pain used, MRI detected abnormalities were highly prevalent in people with (90-97\%) and without (86-88\%) knee pain. While the prevalence of MRI abnormalities was not significantly different in those with versus those without knee pain for most definitions of pain we tested, the prevalence of "any MRI abnormality" was higher in those with WOMAC pain compared with those without pain $(\mathrm{P}=0.002)$. Even so, the prevalence of any MRI abnormality was as high as $86 \%$ in those without WOMAC pain.

\section{Prevalence of bony and soft tissue abnormalities on MRI with more stringent definition}

When we used the more stringent definition of MRI abnormality, overall the prevalence of MRI detected lesions dropped as expected (table $4 \Downarrow$ ), to $14 \%$ for osteophytes, $44 \%$ for cartilage damage, $16 \%$ for bone marrow lesions, $4 \%$ for synovitis, $10 \%$ for attrition, $5 \%$ for subchondral cysts, $8 \%$ for meniscal lesions, and $2 \%$ for ligamentous lesions. The prevalence of any abnormality, however, remained high (53\%, 373/710). Except for bone attrition, painful knees did not differ from those without pain in terms of the prevalence of specific features (table $4 \Downarrow$ ). Regardless of the definition of pain used, any abnormality was present in 57-70\% of participants with pain and about half of those without pain (table $5 \Downarrow$ ). There were significant differences 
between groups with and without pain in three out of four definitions of pain, and the largest difference was seen with WOMAC pain $(15 \%, \mathrm{P}<0.001)$. Prevalence of "any abnormality" in those without WOMAC pain, however, was still high (48\%).

\section{Discussion}

We found that MRI detected features of osteoarthritis are highly prevalent in the tibiofemoral joint of knees that did not have any radiographic features of osteoarthritis in participants both with and without knee pain. Nearly $90 \%$ of our participants had at least one feature of osteoarthritis on MRI. Osteophytes were the most common, followed by cartilage damage and bone marrow lesions. In general, the older the age group, the higher the prevalence of features of osteoarthritis, although differences among age groups were not significant for synovitis and effusion and of borderline significance for ligamentous lesions and bone marrow lesions. Only meniscal lesions were more prevalent in men than women. No significant differences were observed for any type of lesions by BMI.

\section{Strengths and limitations}

This population based study documented the high prevalence of MRI features suggestive of knee osteoarthritis in people without radiographic osteoarthritis. We included only knees that were definitely lacking any radiographic features that could indicate the presence of osteoarthritic changes

(Kellgren-Lawrence grade 0) to ensure our analysis is specific. Although Kellgren-Lawrence grade 1 knees also do not qualify for having radiographic osteoarthritis, a "doubtful" bony abnormality is present and one could argue such equivocal findings are difficult to interpret.

\section{Limitations}

Our sample was primarily (although not exclusively) white, reflecting the population of Framingham, MA. The number of people from other racial or ethnic groups was too small for comparisons. Our prevalence estimates cannot be generalised to adults younger than 50. In particular, meniscal lesions in young active otherwise healthy adults are more likely to be caused by trauma than the degenerative process seen in middle aged and older people. We had no arthroscopic correlation of our MRI findings. Ideally, intra-articular pathology (that is, cartilage, meniscus, and ligaments) should be confirmed by direct visualisation during arthroscopy. Arthroscopy, however, is neither feasible nor ethical in large scale population based studies. Furthermore, arthroscopy cannot visualise some of the MRI findings that are indicative of the osteoarthritis disease process such as subchondral bone marrow lesions. Nearly all the knees in our sample were right knees (with only five left knees). A comparison of 99 people with both right and left knee MRIs in this sample, however, showed no difference in findings, and which knee is studied is therefore unlikely to affect our overall outcome. We did not include the evaluation of radiographic patellofemoral joint pathology in this study because we used the posteroanterior radiograph to classify the tibiofemoral joint of the knee using Kellgren-Lawrence grading. We dealt with this fact by including only subregions of the knee that correspond to the tibiofemoral joint for MRI analysis.

Because we focused on knees with clearly normal radiographic appearance (Kellgren-Lawrence grade 0), we excluded Kellgren-Lawrence grade 1 knees. One might argue that such knees are also without radiographic osteoarthritis and warrant inclusion in our analysis. Inclusion of the 39 Kellgren-Lawrence grade 1 knees in our sample (total 749 knees) did not alter the demographic characteristics of the participants or analytical results for all aspects of the study. There are many ways to define pain, and it is not possible to include all different pain assessment tools available to date in a single study. We selected the WOMAC pain subscale because it has been validated and is widely used..$^{16}$

Our results raise additional questions. More detailed analysis evaluating the factors that could contribute to the differences seen in men and women and the osteoarthritis features in the different age groups would be of interest. Also, comparison of the prevalence of these findings in those with and without radiographic osteoarthritis would tackle the question of whether osteoarthritis is an inevitable consequence of ageing.

\section{Comparison with previous studies}

Of our findings, the most notable is that $74 \%$ of the knees had osteophytes. As a bony abnormality should be clearly visible on radiograph, we did not expect the prevalence to be this high. Presumably, because the MRI assessment used three imaging planes, it could detect osteophytes that were hidden by the overlapping femur or tibia on posteroanterior view radiographs. This is a substantial problem as the presence of definite osteophytes defines the diagnosis of radiographic osteoarthritis. ${ }^{12}$ Thus far, epidemiological or clinical studies of knee osteoarthritis depend largely on the radiographic definition of osteoarthritis. ${ }^{18} 19$ As radiography fails to detect such a large proportion of osteophytes, there could be misclassification of a large number of potentially eligible people in knee osteoarthritis studies and underestimation of the true prevalence of this condition. ${ }^{20}$

Although cartilage itself is aneural and is unlikely to be a direct cause of knee pain, cartilage damage is associated with change in bone marrow lesions, ${ }^{21}$ high BMI, meniscal damage, and synovitis or effusion. ${ }^{22}$ Cartilage thickness has traditionally been assessed by its surrogate marker - the radiographic width of the joint space of the tibiofemoral joint. Narrowing of the joint space, however, can result not only from cartilage damage but also from meniscal lesions. ${ }^{23}$ It has been shown that radiography is less sensitive than MRI for detection of cartilage loss. ${ }^{7}$ Thus, it is not surprising to find a high prevalence of cartilage damage on MRI in the knees of middle aged and older people without radiographic joint space narrowing.

The presence and extent of bone marrow lesions and synovial thickening/effusion can be appreciated only on MRI. These lesions have been associated with pain in knees with osteoarthritis. ${ }^{24}{ }^{25}$ Furthermore, in people at high risk of developing osteoarthritis, bone marrow lesions in asymptomatic knees with no radiographic osteoarthritis at baseline predict development of pain 15 months later. ${ }^{26}$ We also found an association of bone marrow lesions with knee pain among people without radiographic osteoarthritis.

A high prevalence of incidental meniscal findings on MRI in participants of the Framingham Osteoarthritis Study has been reported previously. ${ }^{8}$ One or more meniscal tears was present in $32 \%(41 / 127)$ of knees with symptoms, $23 \%$ (146/548) of knees without symptoms, and $24 \%$ (187/775) overall when there was no or equivocal radiographic evidence of osteoarthritis. Although the results were similar to the present study, they are not identical because Englund and colleagues included knees with Kellgren-Lawrence grade 0 and $1,{ }^{8}$ whereas we focused on Kellgren-Lawrence grade 0 .

We saw fewer incidental ligamentous lesions than any other feature. This could be because the semiquantitative scoring system we used only scores a complete tear as a lesion, and 
partial tears are given a score of zero. Imaging diagnosis of partial ligamentous tears on MRI can be difficult. The role of intra-articular and periarticular ligaments of the knee in predicting structural progression of knee osteoarthritis remains unclear. Disruption of the integrity of these ligaments, however, will probably cause alterations in knee kinematics.

A recent systematic review reported that bone marrow lesions and effusion/synovitis were associated with knee pain. ${ }^{27}$ In our study, however, these lesions were not significantly more prevalent in participants who had knee pain than in those without, with both definitions of MRI abnormality. This discrepancy is probably because the systematic review included only studies involving mostly people with radiographic knee osteoarthritis. Thus, the conclusion of the systematic review is not applicable to the present study.

\section{Clinical implications}

Our findings indicate that the prevalence of MRI detected osteoarthritis features increases with age in the absence of radiographic features of osteoarthritis. We have shown that MRI is more sensitive than radiographs to changes in bone and soft tissue that are considered features of osteoarthritis, ${ }^{28}{ }^{29}$ Our data showed that the prevalence of these MRI detected features is high irrespective of the knee pain status. When we compared the prevalence of MRI abnormalities in knees in people with and without pain, there were two trends. Firstly, and most importantly, the prevalence of MRI findings was extremely high in those without pain, suggesting that using MRI as a diagnostic test for people with normal knee radiographs in this age group would have poor specificity. Secondly, the prevalence of findings was modestly higher in those with pain than in those without, with the difference sometimes reaching significance. These differences, however, were not particularly informative - for example, the highest prevalence of MRI abnormalities was actually in those with mild pain rather than moderate or severe pain.

Thus, MRI features suggestive of osteoarthritis in people without radiographic osteoarthritis are commonly seen in those with or without knee pain, implying that MRI alone is not diagnostically useful to discriminate between people with and without pain in the context of knee osteoarthritis. MRI might still play an important diagnostic role, especially in younger people, in whom other reasons for knee pain should be considered such as inflammatory arthritides, insufficiency fractures, or spontaneous osteonecrosis. Nonetheless, in all likelihood, MRI features of osteoarthritis will be found regardless of the source of the pain. Our study also highlights the limitations of conventional radiography to detect a large number of abnormalities related to osteoarthritis in the knee. ${ }^{20}$

As high BMI is a known risk factor for both incident knee osteoarthritis and for progression of knee osteoarthritis ${ }^{30}$ we expected to see higher prevalence of MRI features in obese people compared with non-obese people. We did not find high BMI to be associated with higher prevalence of MRI features overall compared with low BMI, but rather that these MRI abnormalities were equally highly prevalent in all BMI groups. We speculate that BMI is important for progression of later stages of osteoarthritis, but potentially age is a much more relevant trigger of early stages of osteoarthritis.

Although there is thought to be only a modest correlation between clinical symptoms and radiographic tibiofemoral osteoarthritis, ${ }^{32}$ recent work has highlighted an association between structural osteoarthritis pathology and knee pain. ${ }^{33}{ }^{34}$ It is important for the clinical community to recognise that findings that would be interpreted as abnormal and suggestive of disease are in fact present in most knees without any pain, even when different definitions of pain are used. That means that the clinical significance of these MRI findings is questionable. The same message has been reported for radiographic findings in patients with low back pain (similar highly prevalent abnormalities were seen in those without low back pain), and this led to discouraging radiographic evaluations in those with low back pain. ${ }^{35}$

\section{Conclusions}

Changes indicative of osteoarthritis are commonly present in the knees of most people aged 50 and over who have no radiographic evidence of tibiofemoral osteoarthritis.

Osteophytes, cartilage damage, and bone marrow lesions are especially common among middle aged and older people. These features are common in knees with pain and in those that are painless and can potentially represent pre-radiographic or early stage osteoarthritis. A longitudinal study is needed to determine what proportion of people without radiographic osteoarthritis but with MRI abnormalities subsequently develop radiographic osteoarthritis.

Contributors: AG, JN, DH, and DTF conceived and designed the study. AG, JN, FWR, PA, CEM, and DTF collected the data. AG, DH, FWR, ME, TN, and DTF reviewed the literature. AG, JN, DH, FWR, ME, TN, and DTF directed the analyses, which were carried out by JN. All authors participated in the discussion and interpretation of the results. AG and $\mathrm{DH}$ organised the writing and wrote the initial drafts. All authors critically revised the manuscript for intellectual content and approved the final versions. AG and DTF are guarantors.

Funding: This study was funded by the National Institutes of Health (AG18393 and AR47785) and the Arthritis Foundation. The funders had no role in study design, data collection and analysis, decision to publish, or preparation of the manuscript. The researchers work independently of their funders.

Competing interests: All authors have completed the ICMJE uniform disclosure form at www.icmje.org/coi_disclosure.pdf (available on request from the corresponding author) and declare: $A G$ is the president of Boston Imaging Core Lab (BICL), LLC, and a consultant to Merck Serono, Stryker, Genzyme, AstraZeneca, and Novartis; FWR a vice president and shareholder of BICL and is a consultant to Merck Serono and National Institute of Health; ME is funded by the Swedish Research Council, the Greta and Johan Kock Foundation, King Gustaf V 80-year Birthday Foundation, and the Faculty of Medicine, Lund University, Sweden; TN is supported by NIAMS AR055127 and the Arthritis Foundation Arthritis Investigator Award.

Ethical approval: This study was approved by the institutional review board of Boston University Medical Centre (protocol number H-22674), and written informed consent was obtained from all participants.

Data sharing: No additional data available.

1 Sacks JJ, Luo YH, Helmick CG. Prevalence of specific types of arthritis and other rheumatic conditions in the ambulatory health care system in the United States, 2001-2005. Arthritis Care Res (Hoboken) 2010;62:460-4.

2 Bedson J, Jordan K, Croft P. The prevalence and history of knee osteoarthritis in general practice: a case-control study. Fam Pract 2005;22:103-8.

3 Murphy L, Schwartz TA, Helmick CG, Renner JB, Tudor G, Koch G, et al. Lifetime risk of symptomatic knee osteoarthritis. Arthritis Rheum 2008:59:1207-13.

4 Wills AK, Black S, Cooper R, Coppack RJ, Hardy R, Martin KR, et al. Life course body mass index and risk of knee osteoarthritis at the age of 53 years: evidence from the 1946 British birth cohort study. Ann Rheum Dis 2012;71:655-60.

5 Felson DT, Zhang Y. An update on the epidemiology of knee and hip osteoarthritis with a view to prevention. Arthritis Rheum 1998:41:1343-55.

6 Lawrence RC, Felson DT, Helmick CG, Arnold LM, Choi H, Deyo RA, et al. Estimates of the prevalence of arthritis and other rheumatic conditions in the United States. Part II. Arthritis Rheum 2008;58:26-35.

7 Amin S, LaValley MP, Guermazi A, Grigoryan M, Hunter DJ, Clancy M, et al. The relationship between cartilage loss on magnetic resonance imaging and radiographic progression in men and women with knee osteoarthritis. Arthritis Rheum 2005;52:3152-9. 


\section{What is already known on this topic}

MRI can detect features suggestive of knee osteoarthritis that cannot be visualised on conventional radiography, which is insensitive to many findings

In roughly half of people with knee pain, radiography shows no abnormalities

\section{What this study adds}

Changes indicative of osteoarthritis are commonly present in the knees of most people aged 50 and over who have no radiographic evidence of tibiofemoral osteoarthritis

MRI detected findings of osteoarthritis are common in people with and without knee pain, suggesting that the clinical significance of MRI findings in such knees is not clear

8 Englund M, Guermazi A, Gale D, Hunter DJ, Aliabadi P, Clancy M, et al. Incidental meniscal findings on knee MRI in middle-aged and elderly persons. N Engl J Med 2008;359:1108-15.

9 Vernooij MW, Ikram MA, Tanghe HL, Vincent AJ, Hofman A, Krestin GP, et al. Incidental findings on brain MRI in the general population. N Engl J Med 2007;357:1821-8.

10 Guermazi A, Roemer FW, Hayashi D. Imaging of osteoarthritis: update from a radiological perspective. Curr Opin Rheumatol 2011;23:484-91.

11 Kothari M, Guermazi A, von Ingersleben G, Miaux Y, Sieffert M, Block JE, et al. Fixed-flexion radiography of the knee provides reproducible joint space width measurements in osteoarthritis. Eur Radiol 2004;14:1568-73.

12 Kellgren JH, Lawrence JS. Radiological assessment of osteo-arthrosis. Ann Rheum Dis 1957;16:494-502.

13 Felson DT, Naimark A, Anderson J, Kazis L, Castelli W, Meenan RF. The prevalence of knee osteoarthritis in the elderly. The Framingham Osteoarthritis Study. Arthritis Rheum 1987;30:914-8.

14 Peterfy CG, Guermazi A, Zaim S, Tirman PF, Miaux Y, White D, et al. Whole-organ magnetic resonance imaging score (WORMS) of the knee in osteoarthritis. Osteoarthritis Cartilage 2004:12:177-90.

15 Roemer FW, Frobell R, Hunter DJ, Crema MD, Fischer W, Bohndorf K, et al. MRI-detected subchondral bone marrow signal alterations of the knee joint: terminology, imaging appearance, relevance and radiological differential diagnosis. Osteoarthritis Cartilage 2009;17:1115-31.

16 Theiler R, Sangha O, Schaeren S, Michel BA, Tyndall A, Dick W, et al. Superior responsiveness of the pain and function sections of the Western Ontario and McMaster Universities Osteoarthritis Index (WOMAC) as compared to the Lequesne-Algofunctional Index in patients with osteoarthritis of the lower extremities. Osteoarthritis Cartilage 1999; $7: 515-9$

17 Avasthi S, Sanghi D, Singh A, Kumar A, Kumar S, Misra A, et al. Significance of clinical parameters and role of clinical scoring systems in predicting severity of primary osteoarthritis knee. Int J Orthoped Surg 2009:13:1.

18 Felson DT, Gale DR, Elon Gale M, Niu J, Hunter DJ, Goggins J, et al. Osteophytes and progression of knee osteoarthritis. Rheumatology (Oxford) 2005;44:100-4.

19 Guermazi A, Hunter DJ, Li L, Benichou O, Eckstein F, Kwoh CK, et al. Different thresholds for detecting osteophytes and joint space narrowing exist between the site investigators and the centralized reader in a multicenter knee osteoarthritis study-data from the Osteoarthritis Initiative. Skeletal Radiol 2012;41:179-86.

20 Sanghi D, Avasthi S, Mishra A, Singh A, Agarwal S, Srivastava RN. Is radiology a determinant of pain, stiffness, and functional disability in knee osteoarthritis? A cross-sectional study. J Orthop Sci 2011;16:719-25.

21 Roemer FW, Guermazi A, Javaid MK, Lynch JA, Niu J, Zhang Y, et al. Change in MRI-detected subchondral bone marrow lesions is associated with cartilage loss: the MOST Study. A longitudinal multicentre study of knee osteoarthritis. Ann Rheum Dis 2009:68:1461-5.

22 Roemer FW, Zhang Y, Niu J, Lynch JA, Crema MD, Marra MD, et al. Tibiofemoral joint osteoarthritis: risk factors for MR-depicted fast cartilage loss over a 30 -month period in the multicenter osteoarthritis study. Radiology 2009;252:772-80.
23 Hunter DJ, Zhang YQ, Tu X, Lavalley M, Niu JB, Amin S, et al. Change in joint space width: hyaline articular cartilage loss or alteration in meniscus? Arthritis Rheum 2006;54:2488-95.

24 Felson DT, Chaisson CE, Hill CL, Totterman SM, Gale ME, al. SKe. The association of bone marrow lesions with pain in knee osteoarthritis. Ann Intern Med 2001:134:541-9.

25 Hill CL, Gale DG, Chaisson CE, Skinner K, Kazis L, Gale ME, et al. Knee effusions, popliteal cysts, and synovial thickening: association with knee pain in osteoarthritis. $J$ Rheumatol 2001;28:1330-7.

26 Javaid MK, Lynch JA, Tolstykh I, Guermazi A, Roemer F, Aliabadi P, et al. Pre-radiographic MRI findings are associated with onset of knee symptoms: the most study. Osteoarthritis Cartilage 2010;18:323-8.

27 Yusuf E, Kortekaas MC, Watt I, Huizinga TW, Kloppenburg M. Do knee abnormalities visualised on MRI explain knee pain in knee osteoarthritis? A systematic review. Ann Rheum Dis 2011;70:60-7.

28 Peterfy CG, Gold G, Eckstein F, Cicuttini F, Dardzinski B, Stevens R. MRI protocols for whole-organ assessment of the knee in osteoarthritis. Osteoarthritis Cartilage 2006;14(suppl A):A95-111.

29 Conaghan PG, Felson D, Gold G, Lohmander S, Totterman S, Altman R. MRI and non-cartilaginous structures in knee osteoarthritis. Osteoarthritis Cartilage 2006;14(suppl A):A87-94.

30 Neogi T, Zhang Y. Osteoarthritis prevention. Curr Opin Rheumatol 2011;23:185-91.

31 Yusuf E, Bijsterbosch J, Slagboom PE, Rosendaal FR, Huizinga TW, Kloppenburg M. Body mass index and alignment and their interaction as risk factors for progression of knees with radiographic signs of osteoarthritis. Osteoarthritis Cartilage 2011;19:1117-22.

32 Szebenyi B, Hollander AP, Dieppe P, Quilty B, Duddy J, Clarke S, et al. Associations between pain, function, and radiographic features in osteoarthritis of the knee. Arthritis Rheum 2006;54:230-5.

33 Zhang Y, Nevitt M, Niu J, Lewis C, Torner J, Guermazi A, et al. Fluctuation of knee pain and changes in bone marrow lesions, effusions, and synovitis on magnetic resonance imaging. Arthritis Rheum 2011;63:691-9.

34 Neogi T, Felson D, Niu J, Nevitt M, Lewis CE, Aliabadi P, et al. Association between radiographic features of knee osteoarthritis and pain: results from two cohort studies. BMJ 2009;339:b2844.

35 Chou R, Fu R, Carrino JA, Deyo RA. Imaging strategies for low-back pain: systematic review and meta-analysis. Lancet 2009;373:463-72.

Accepted: 23 July 2012

\section{Cite this as: BMJ 2012;345:e5339}

This is an open-access article distributed under the terms of the Creative Commons Attribution Non-commercial License, which permits use, distribution, and reproduction in any medium, provided the original work is properly cited, the use is non commercial and is otherwise in compliance with the license. See: http://creativecommons.org/licenses/by$\mathrm{nc} / 2.0 /$ and http://creativecommons.org/licenses/by-nc/2.0/legalcode. 


\section{Tables}

Table 1/ Characteristics of participants without knee abnormalities. Figures are numbers (percentage) unless stated otherwise

\begin{tabular}{lc}
$\begin{array}{lc}\text { Characteristic } \\
\text { Age (years): }\end{array}$ & Data $(\mathbf{n}=\mathbf{7 1 0})$ \\
\hline Mean (SD) & $62.3(8.4)$ \\
\hline Range & $(51-89)$ \\
\hline$\geq 50-<60$ & $316(45)$ \\
\hline$\geq 60-<70$ & $249(35)$ \\
\hline$\geq 70$ & $145(20)$ \\
\hline Body mass index (BMI): \\
\hline Mean (SD) & $27.9(5.1)$ \\
\hline Range & $(16.6-50.6)$ \\
\hline$<25.0$ & $222(31)$ \\
\hline $25-29.9$ & $278(39)$ \\
\hline$\geq 30$ & $204(29)$ \\
\hline Women & $393(55)$ \\
\hline White & $660(93)$ \\
\hline Knee pain present & $206(29)$
\end{tabular}


Table 2| Prevalence of MRI features (standard definition*) stratified by sex, pain status, and BMI. Figures are numbers (percentage) of participants

\begin{tabular}{|c|c|c|c|c|c|c|c|c|c|c|c|}
\hline \multirow[b]{2}{*}{ MRI features } & \multirow[b]{2}{*}{$\begin{array}{l}\text { Overall } \\
(n=710)\end{array}$} & \multicolumn{3}{|c|}{ Sex } & \multicolumn{3}{|c|}{ Knee pain } & \multicolumn{4}{|c|}{ BMI } \\
\hline & & $\begin{array}{l}\text { Women } \\
(n=393)\end{array}$ & $\begin{array}{c}\text { Men } \\
(n=317)\end{array}$ & $P$ value & $\begin{array}{c}\text { Pain } \\
(n=206)\end{array}$ & $\begin{array}{l}\text { No pain } \\
(n=489)\end{array}$ & $P$ value & $\begin{array}{c}<25.0 \\
(n=222)\end{array}$ & $\begin{array}{l}25-29.9 \\
(n=278)\end{array}$ & $\begin{array}{c}\geq 30 \\
(n=204)\end{array}$ & $P$ value \\
\hline $\begin{array}{l}\text { Any } \\
\text { abnormality }\end{array}$ & $631(89)$ & $346(88)$ & $285(90)$ & 0.43 & $188(91)$ & $430(88)$ & 0.20 & $193(87)$ & $249(90)$ & $184(90)$ & 0.51 \\
\hline Osteophytes & $524(74)$ & $281(72)$ & $243(77)$ & 0.12 & $158(77)$ & $353(72)$ & 0.22 & $154(69)$ & $208(75)$ & $157(77)$ & 0.18 \\
\hline $\begin{array}{l}\text { Cartilage } \\
\text { damage }\end{array}$ & $492(69)$ & $273(70)$ & $219(69)$ & 0.91 & $149(72)$ & $333(68)$ & 0.27 & $153(69)$ & $195(70)$ & $139(68)$ & 0.89 \\
\hline $\begin{array}{l}\text { Bone marrow } \\
\text { lesions }\end{array}$ & $371(52)$ & $213(54)$ & $158(50)$ & 0.25 & $121(59)$ & $242(50)$ & 0.03 & $117(53)$ & $149(54)$ & $103(51)$ & 0.79 \\
\hline Synovitis & $259(37)$ & $139(35)$ & $120(38)$ & 0.49 & $78(38)$ & $175(36)$ & 0.60 & $88(40)$ & $99(36)$ & $69(34)$ & 0.43 \\
\hline Attrition & $228(32)$ & $124(32)$ & $104(33)$ & 0.72 & $78(38)$ & $147(30)$ & 0.04 & $84(38)$ & $79(28)$ & $63(31)$ & 0.07 \\
\hline $\begin{array}{l}\text { Subchondral } \\
\text { cysts }\end{array}$ & $179(25)$ & $108(28)$ & $71(22)$ & 0.12 & $63(31)$ & $114(23)$ & 0.04 & $59(27)$ & $69(25)$ & $50(25)$ & 0.86 \\
\hline $\begin{array}{l}\text { Meniscal } \\
\text { lesions }\end{array}$ & $167(24)$ & $57(15)$ & $110(35)$ & $<0.001$ & $42(20)$ & $120(25)$ & 0.24 & $56(25)$ & $72(26)$ & $38(19)$ & 0.14 \\
\hline $\begin{array}{l}\text { Ligamentous } \\
\text { lesions }\end{array}$ & $66(9)$ & $31(8)$ & $35(11)$ & 0.15 & $22(11)$ & $43(9)$ & 0.44 & $17(8)$ & $25(9)$ & $23(11)$ & 0.43 \\
\hline
\end{tabular}

*Cartilage lesions and osteophytes=grade $\geq 2$; all other lesions=grade $\geq 1$; "any abnormality"=presence of any type of abnormality as defined above. All MRI detected lesions graded with whole organ magnetic resonance imaging score (WORMS). 
Table 3| Prevalence of MRI features (standard definition ${ }^{\star}$ ) stratified by age group. Figures are numbers (percentage) of participants

\begin{tabular}{|c|c|c|c|c|}
\hline \multirow[b]{2}{*}{ MRI features } & \multicolumn{4}{|c|}{ Age group (years) } \\
\hline & $\geq 50-<60(n=316)$ & $\geq 60-<70(n=249)$ & $\geq 70(n=145)$ & $P$ value \\
\hline Osteophytes & $68(215 / 316)$ & $78(193 / 249)$ & $80(116 / 145)$ & 0.007 \\
\hline Cartilage damage & $61(193 / 316)$ & $74(183 / 249)$ & $80(116 / 145)$ & $<0.001$ \\
\hline Bone marrow lesions & $48(150 / 316)$ & $55(137 / 249)$ & $58(84 / 145)$ & 0.06 \\
\hline Synovial thickening and joint effusion & $32(102 / 316)$ & $39(97 / 249)$ & $41(60 / 145)$ & 0.10 \\
\hline Attrition & $29(91 / 316)$ & $35(86 / 249)$ & $35(51 / 145)$ & 0.20 \\
\hline Subchondral cysts & $19(61 / 316)$ & $29(71 / 249)$ & $32(47 / 145)$ & 0.004 \\
\hline Meniscal lesions & $17(55 / 316)$ & $24(60 / 249)$ & $36(52 / 145)$ & $<0.001$ \\
\hline Ligamentous lesions & $7(22 / 316)$ & $10(24 / 249)$ & $14(20 / 145)$ & 0.06 \\
\hline
\end{tabular}

${ }^{*}$ Cartilage lesions and osteophytes $=$ grade $\geq 2$; all other lesions=grade $\geq 1$. 
Table 4| Prevalence of MRI features (more stringent definition ${ }^{\star}$ ) stratified by sex, pain status, and BMI. Figures are numbers (percentage) of participants

\begin{tabular}{|c|c|c|c|c|c|c|c|c|c|c|c|}
\hline \multirow[b]{2}{*}{ MRI features } & \multirow[b]{2}{*}{$\begin{array}{l}\text { Overall } \\
(n=710)\end{array}$} & \multicolumn{3}{|c|}{ Sex } & \multicolumn{3}{|c|}{ Knee pain $†$} & \multicolumn{4}{|c|}{ BMI } \\
\hline & & $\begin{array}{l}\text { Women } \\
(n=393)\end{array}$ & $\begin{array}{c}\text { Men } \\
(n=317)\end{array}$ & $P$ value & $\begin{array}{c}\text { Pain } \\
(n=206)\end{array}$ & $\begin{array}{l}\text { No pain } \\
(n=489)\end{array}$ & $P$ value & $\begin{array}{c}<25.0 \\
(n=222)\end{array}$ & $\begin{array}{l}25-29.9 \\
(n=278)\end{array}$ & $\begin{array}{c}\geq 30 \\
(n=204)\end{array}$ & $P$ value \\
\hline Any abnormality & $373(53)$ & $198(50)$ & $175(55)$ & 0.20 & $118(57)$ & $249(51)$ & 0.13 & $116(52)$ & $145(52)$ & $108(53)$ & 0.98 \\
\hline Osteophytes & $98(14)$ & $47(12)$ & $51(16)$ & 0.11 & $31(15)$ & $65(13)$ & 0.54 & $22(10)$ & $39(14)$ & $36(18)$ & 0.07 \\
\hline $\begin{array}{l}\text { Cartilage } \\
\text { damage }\end{array}$ & $309(44)$ & $174(44)$ & $135(43)$ & 0.65 & $97(47)$ & $208(43)$ & 0.27 & $98(44)$ & $118(43)$ & $90(44)$ & 0.91 \\
\hline $\begin{array}{l}\text { Bone marrow } \\
\text { lesions }\end{array}$ & $115(16)$ & $66(17)$ & $49(16)$ & 0.63 & 39 (19) & $75(15)$ & 0.24 & $33(15)$ & $43(16)$ & $37(18)$ & 0.62 \\
\hline Synovitis & $31(4)$ & $11(3)$ & $20(6)$ & 0.02 & $10(5)$ & $21(4)$ & 0.74 & $9(4)$ & $11(4)$ & $10(5)$ & 0.86 \\
\hline Attrition & $7010)$ & $41(10)$ & $29(9)$ & 0.57 & $29(14)$ & $39(8)$ & 0.01 & $24(11)$ & $24(9)$ & $21(10)$ & 0.69 \\
\hline $\begin{array}{l}\text { Subchondral } \\
\text { cysts }\end{array}$ & $32(5)$ & $19(5)$ & $13(4)$ & 0.64 & $11(5)$ & $21(4)$ & 0.55 & $11(5)$ & $16(6)$ & $5(2)$ & 0.21 \\
\hline $\begin{array}{l}\text { Meniscal } \\
\text { lesions }\end{array}$ & $58(8)$ & $19(5)$ & $39(12)$ & $<0.001$ & $18(9)$ & $40(8)$ & 0.81 & $23(10)$ & $23(8)$ & $12(6)$ & 0.24 \\
\hline $\begin{array}{l}\text { Ligamentous } \\
\text { lesions }\end{array}$ & $15(2)$ & $3(1)$ & $12(4)$ & 0.005 & $5(2)$ & $10(2)$ & 0.75 & $5(2)$ & $6(2)$ & $4(2)$ & 0.98 \\
\hline
\end{tabular}

${ }^{*}$ Cartilage damage and osteophytes=WORMS grade $\geq 3$; all other lesions=grade $\geq 2$.

†Presence of knee pain was assessed by question: "In the past month, have you had any pain, aching or stiffness." 
Table 5| Prevalence of "any abnormality" on MRI stratified by pain status with standard and more stringent definitions of pain. Figures are numbers (percentage) of participants

\begin{tabular}{|c|c|c|c|c|}
\hline Pain definition* & Pain present & Mild pain & No pain & $P$ value for difference \\
\hline \multicolumn{5}{|c|}{ Presence of knee pain in past month†: } \\
\hline Standard definition & 188/206 (91) & - & $430 / 489(88)$ & 0.20 \\
\hline More stringent definition & $118 / 206(57)$ & - & $249 / 489(51)$ & 0.13 \\
\hline \multicolumn{5}{|c|}{ Knee pain $\ddagger$ lasting at least a month in past yeart: } \\
\hline Standard definition & 90/98 (92) & $54 / 56(96)$ & $474 / 541(88)$ & 0.08 \\
\hline More stringent definition & $57 / 98(58)$ & $39 / 56(70)$ & $272 / 541(50)$ & 0.01 \\
\hline \multicolumn{5}{|l|}{ Knee pain $\ddagger$ on most days $\dagger$ : } \\
\hline Standard definition & $45 / 50(90)$ & $58 / 60(97)$ & $516 / 586(88)$ & 0.12 \\
\hline More stringent definition & $29 / 50(58)$ & $41 / 60(68)$ & $297 / 586(51)$ & 0.02 \\
\hline \multicolumn{5}{|c|}{ Score $\geq 1$ in any WOMAC pain questions in person with Kellgren Lawrence grade 0 knees bilaterally§: } \\
\hline Standard definition & $219 / 233(94)$ & - & $411 / 476(86)$ & 0.002 \\
\hline More stringent definition & $146 / 233(63)$ & - & $226 / 476(48)$ & $<0.001$ \\
\hline
\end{tabular}

*Standard definition includes cartilage lesions and osteophytes=grade $\geq 2$; all other lesions=grade $\geq 1$; any abnormality=presence of any type of abnormality as defined above. More stringent definition includes cartilage damage and osteophytes=WORMS grade $\geq 3$; all other lesions=grade $\geq 2$.

†Knee level pain assessment.

¥Moderate/severe pain.

§Person level pain assessment. 


\section{Figures}

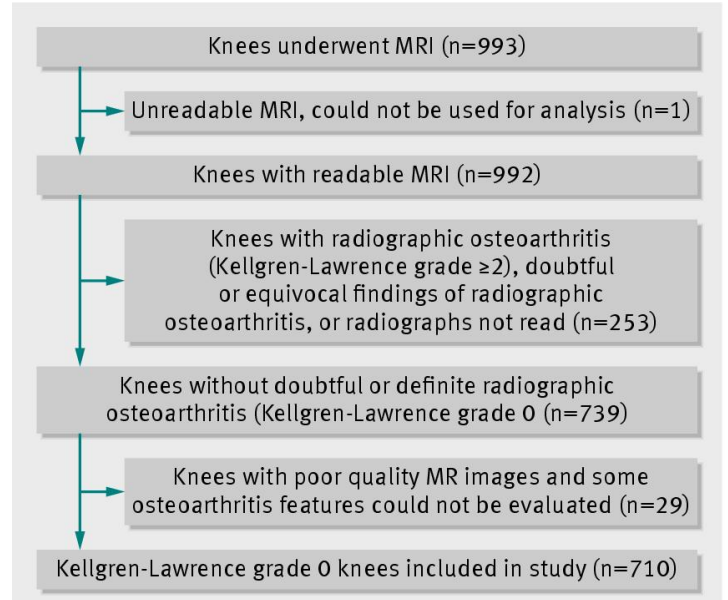

Fig 1 Selection process of knees included in present study


Fig 2 Knee with multiple abnormalities on MRI indicating early stage osteoarthritis despite lack of radiographic osteoarthritis. A: coronal fat suppressed proton density weighted image shows several features of early OA detectable only by MRI. White arrowhead shows focal full thickness cartilage defect at central weight bearing part of medial femur. In addition there is adjacent subchondral bone marrow lesion presenting as area of ill defined hyperintensity (arrows). Black arrowheads show meniscal extrusion at medial joint line causing bulging of neighbouring medial collateral ligament (no arrow). B: sagittal proton density weighted image shows isolated degenerative horizontal oblique tear of posterior horn of medial meniscus extending to undersurface of meniscus adjacent to posterior tibial surface (arrows). No associated cartilage damage or subchondral bony alterations are seen 


\begin{tabular}{lcc} 
& \multicolumn{2}{c}{ P value } \\
\cline { 2 - 3 } & $\begin{array}{c}\text { Standard } \\
\text { definition }\end{array}$ & $\begin{array}{c}\text { More stringent } \\
\text { definition }\end{array}$ \\
- Any abnormality & 0.06 & $<0.001$ \\
- - - Osteophytes & 0.007 & 0.01 \\
--- Cartilage damage & $<0.001$ & 0.001 \\
-- - Bone marrow lesion & 0.06 & 0.33 \\
- - Synovitis & 0.10 & 0.01 \\
- - Attrition & 0.24 & 0.003 \\
-.- - Subchondral cyst & 0.004 & 0.06 \\
--- - Meniscal damage & $<0.001$ & $<0.001$ \\
- - - Ligamentous damage & 0.06 & 0.43
\end{tabular}
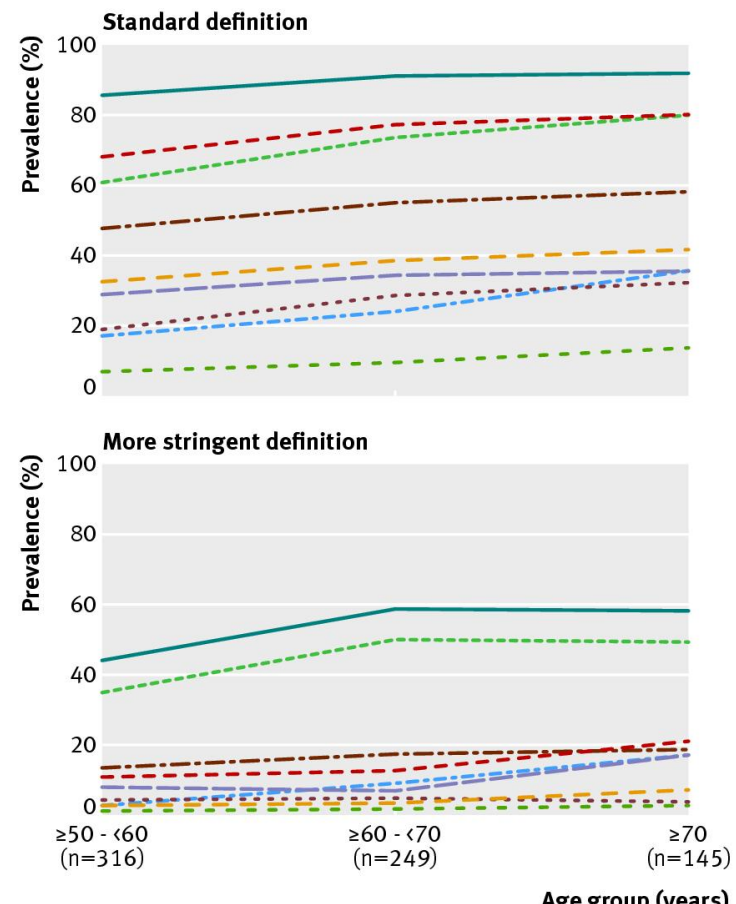

Fig 3 Prevalence of osteoarthritis features on MRI in knees without radiographic osteoarthritis stratified by age group with standard and more stringent definitions of MRI abnormalities 